\title{
A Diagnosis-Prognosis Feedback Loop for Improved Performance Under Uncertainties
}

\author{
Patrick E. Leser* and James E. Warner ${ }^{\dagger}$ \\ NASA Langley Research Center, Hampton, VA, 23666, USA.
}

\begin{abstract}
The feed-forward relationship between diagnosis and prognosis is the foundation of both aircraft structural health management and the digital twin concept. Measurements of structural response are obtained either in-situ with mounted sensor networks or offline using more traditional techniques (e.g., nondestructive evaluation). Diagnosis algorithms process this information to detect and quantify damage and then feed this data forward to a prognostic framework. A prognosis of the structure's future operational readiness (e.g., remaining useful life or residual strength) is then made and is used to inform missioncritical decision-making. Years of research have been devoted to improving the elements of this process, but the process itself has not changed significantly. Here, a new approach is proposed in which prognosis information is not only fed forward for decision-making, but it is also fed back to the forthcoming diagnosis. In this way, diagnosis algorithms can take advantage of a priori information about the expected state of health, rather than operating in an uninformed condition. As a feasibility test, a diagnosis-prognosis feedback loop of this manner is demonstrated. The approach is applied to a numerical example in which fatigue crack growth is simulated in a simple aluminum alloy test specimen. A prognosis was derived from a set of diagnoses which provided feedback to a subsequent set of diagnoses. Improvements in accuracy and a reduction in uncertainty in the prognosisinformed diagnoses were observed when compared with an uninformed diagnostic approach.
\end{abstract}

\section{Introduction}

In the context of structural health management (SHM), digital twin focuses on the coupling of highfidelity life predictions with in-situ damage diagnostics. ${ }^{1,2}$ These two concepts are often considered as separate entities. Damage diagnosis is an active area of research in which changes in modal properties, strain response, or guided wave scattering patterns are used to detect and quantify flaws or defects in a structure. Damage diagnosis is a process that involves multiple scales (i.e., damage is manifested at the microstructural level, and measurements are often taken at the macroscale, sometimes at large distances away). Uncertainties are introduced to the problem at each scale and via models used to form the diagnoses; therefore, quantifying these uncertainties is paramount if the gathered data is to be used for decision-making. ${ }^{3}$ Damage prognosis is typically a two-step process that can also encompass multiple scales and suffers from multi-source uncertainty. To form a prognosis, the uncertainties in both the set of available diagnoses and the parameters of a chosen predictive model must be quantified. These uncertainties are then propagated through the model to produce a probabilistic prediction of remaining useful life (RUL). ${ }^{3}$

Significant work has been invested in developing the relationship between damage diagnostics and prognostics. For example, Sankararaman et al. developed a prognosis framework to interface with damage diagnosis data and used it to predict fatigue crack growth in an aluminum cylinder with quantified uncertainties. ${ }^{4,5}$ Peng et al. used Markov chain Monte Carlo (MCMC) sampling methods to combine diagnosis, prognosis and uncertainty quantification for an aluminum lap joint. ${ }^{6}$ Similar methods have been applied to composite materials as well, as demonstrated by the particle filtering approach used by Chiachio et al. ${ }^{7}$ to predict fatigue delamination growth. These are only a small sampling from the field, but all of these works share a common thread in that the relationship between diagnosis and prognosis is unidirectional. Furthermore, most approaches tend to treat the diagnostic problem as deterministic.

\footnotetext{
* Research Student Trainee (Engineering); Durability, Damage Tolerance, and Reliability Branch.

${ }^{\dagger}$ Research Computer Scientist; Durability, Damage Tolerance, and Reliability Branch.
} 
As uncertainty quantification becomes more prevalent in damage diagnostics research, an opportunity arises in which the current linear relationship between diagnosis and prognosis can be evolved into a more complex, cyclical relationship. More precisely, a feedback loop can be developed with prognosis feeding information about expected future damage conditions back to the diagnosis framework, as shown in Figure 1. If the uncertainty quantification method used for diagnosis is based on a Bayesian ideology, then this information would take the form of a prior distribution. In situations where noise levels or lack of data inhibit Bayesian inference of the damage parameters, an informed prior distribution can allow for successful damage diagnosis. It should be noted that the overarching feedback concept could be extended to a more informed, experimental diagnostic approach as well. For example, when using guided wave-based diagnostics, the transmission and reflectivity ratios are highly dependent on the type and size of damage as well as the frequency of the impending wave. ${ }^{8,9}$ A prognosis of the damage state at the time the next measurement is to be taken could allow for tuning of the excitation frequencies and a potential improvement of image resolution or probability of detection. The work presented herein focuses primarily on the idea of stochastic feedback, and experimental applications such as this are left for future work.

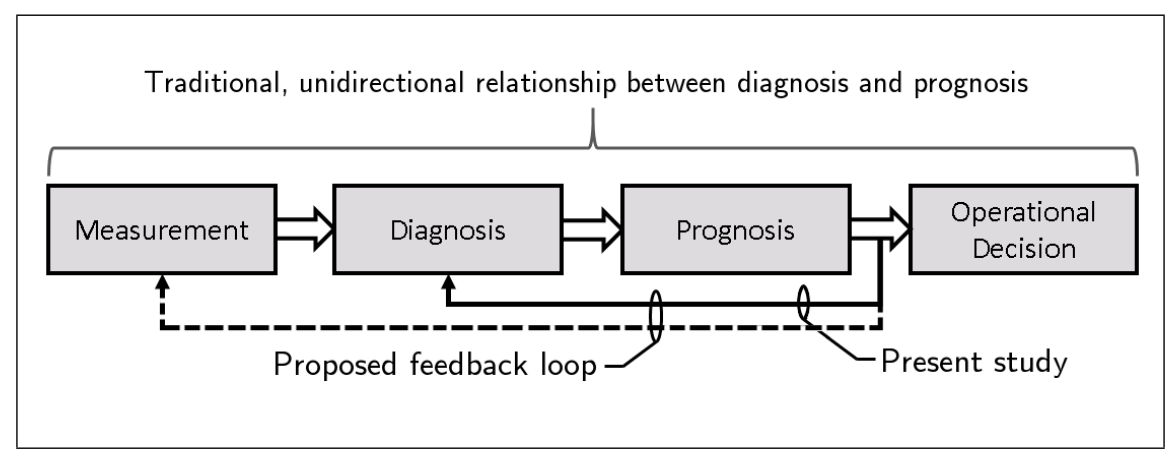

Figure 1. Diagram of the proposed feedback loop. While feedback can be applied to measurement techniques as well as the diagnostic framework, the present work is only focused on providing stochastic feedback to the diagnosis.

The hypothesis for the present study was formulated as follows: prognosis of the damage state at a future time instant can inform a future diagnosis made at that same time instant, resulting in an improvement of accuracy and a reduction in uncertainty. The feedback was assumed to be stochastic in nature, taking the form of a prior distribution fed to the Bayesian diagnostic framework. The work was intended to be a feasibility study; as such, to control for excessive randomness from model or experimental errors, a numerical fatigue crack growth simulation was conducted using finite element analysis (FEA). A set of diagnoses (i.e., strain-based estimates of crack length over time) were used to produce a probabilistic estimate of the crack length at future time instants corresponding to planned diagnoses. These predicted distributions of crack lengths were fed to the diagnostic framework as prior distributions, and the final diagnoses were made. To test the effect of the feedback loop, a diagnosis was also made with a noninformative prior distribution at each examined time. The accuracy and uncertainty of the two results were then compared relative to the ground truth simulation.

\section{Formulation}

Let the useful life of a structure be discretized by the times $t_{i}$, where $i=1, \ldots, p, \ldots E O L$. Damage prognosis involves making a diagnosis at each index up to $t_{p}$, the time at which a prediction will be made. At this time, the diagnosis information for all past time steps are gathered and used to quantify the uncertainties in both the measurements and the predictive model. These uncertainties are propagated forward in time to some end of life (EOL), or the time at which failure is deemed to have occurred, $t_{E O L}$. This concept is integral to both digital twin and SHM, as discussed in the introduction. However, predictive information from the time between $t_{p}$ and $t_{E O L}$ is often unused or discarded. Here, it is proposed that this information be utilized to inform the next damage diagnosis measurement at time $t_{p}+\tau$, where $\tau$ is an arbitrary step forward in time. For any $\tau$, a distribution of predicted damage states is available that can be recast as a prior distribution for an upcoming diagnosis to be made at $t_{p}+\tau$.

Completing the feedback loop from prognosis back to diagnosis in this manner has the potential to enhance the identifiability of damage in a model-based (inverse problem) SHM approach. A primary challenge of such 
methods is that the inverse problem is typically ill-posed, ${ }^{10}$ e.g., a possibly infinite number of damage states can reproduce the sensor data. This problem is exacerbated with decreased signal-to-noise ratio resulting from sensor error, sparse measurements, small damage size, and limited sensor proximity to damage. In deterministic approaches to inverse problem SHM, a carefully-tuned regularization strategy must be employed during the solution process to ensure a unique solution exists. ${ }^{11}$ Under a Bayesian regime, regularization is carried out more naturally through the specification of an informative prior distribution, favoring those solutions to the inverse problem that agree with any available a priori information, including expert opinion and results from previous analyses. While prior distributions for the location of damage initiation can be developed from offline hot spot identification, any reasonable prior distribution for damage size or severity must be generated online using prognostics.

In a general SHM Bayesian inference problem, a parameter-dependent model response, $f(Q)$, experimental measurements, $\Upsilon$, and measurement errors, $\varepsilon$, are related through the statistical model

$$
\Upsilon=f(Q)+\varepsilon .
$$

Here, $Q, \varepsilon$, and $\Upsilon$ are random variables. The variable $Q$ denotes the model parameters and has realizations $q$ while $\Upsilon$ has realizations in the form of observations, denoted by $v$. For diagnostics, the model response would be a directly measurable quantity like dynamic response or strains, and the parameters would characterize the damage in a given structure or component. For prognostics, the model response would be of the same form as the diagnostic output, and the parameters would be input to some predictive model (e.g., crack growth rate parameters). The goal of the Bayesian inverse problem in the context of SHM is to determine the posterior density, $\pi\left(q \mid v_{o b s}\right)$, that reflects the best or most likely distribution of parameter values based on the available observations. The multi-variate parameter distribution can be determined using Bayes' Theorem,

$$
\pi(q \mid v)=\frac{\pi(v \mid q) \pi_{0}(q)}{\pi(v)}=\frac{\pi(v \mid q) \pi_{0}(q)}{\int_{\mathbb{R}^{p}} \pi(v \mid q) \pi_{0}(q) d q} .
$$

While the details will be omitted for brevity, obtaining a direct solution for the posterior in Equation 2 is often intractable due to the evaluation of the normalizing constant in the denominator. However, sampling techniques exist ${ }^{12}$ that enable the approximation of the posterior given the likelihood distribution, $\pi(v \mid q)$, (determined through a comparison of model responses and observations) and the prior distribution, $\pi_{0}(q)$, of the parameters. To avoid influencing the inverse problem with inaccurate prior knowledge, it is common practice to use a noninformative prior distribution; i.e., an improper uniform density over a bounded support. ${ }^{12,13}$ However, as mentioned above, there are significant benefits to an informative prior distribution. To reap these benefits, the prior distribution at a given time after a prognosis has been made, $t=t_{p}+\tau$, is

$$
\pi_{0}\left(q, t_{p}+\tau\right)=\mathcal{P}\left(\theta, t_{p}+\tau, \mathbb{D}_{t_{p}}\right),
$$

where $\mathcal{P}$ is the stochastic response of the predictive model extrapolated forward in time by $\tau$ given a set of model parameters, $\theta$, and the set of damage diagnoses available at the time of prediction, $\mathbb{D}_{t_{p}}$.

\section{Numerical Example}

In this work, a numerical example is used to demonstrate the diagnosis-prognosis coupling. An aluminum alloy 2024-T3 tensile specimen was modeled using finite elements, ${ }^{14}$ and the fracture mechanics code FRANC3D ${ }^{15}$ was used to insert a through-crack of length 0.0735 in into the model and simulate its growth under constant amplitude fatigue loading. The maximum applied stress was $6.25 \mathrm{ksi}$ and the minimum applied stress was $0.625 \mathrm{ksi}$. The crack was grown using linear elastic fracture mechanics and a median extension approach. ${ }^{15}$ An illustration of the specimen and associated boundary conditions is shown in Figure 2. Additional information regarding the model can be found in the Appendix.

Stress intensity factors (SIFs), denoted as $K$, were computed via finite element analysis of the cracked mesh and used to define the vector of crack extensions over the discretized crack front. The crack was extended at each step such that the median growth was equal to a user-defined value of 0.005 in. The crack growth rate, $\frac{d a}{d t}$, was defined using the NASGRO (version 4) equation ${ }^{16}$ as 


$$
\frac{d a}{d t}=C\left[\left(\frac{1-f}{1-R}\right) \Delta K\right]^{n} \frac{\left(1-\frac{\Delta K_{t h}}{\Delta K}\right)^{p}}{\left(1-\frac{K_{\max }}{K_{C}}\right)^{q}},
$$

with units of in/cycle. The parameters $C, n, p$, and $q$ are empirical constants. The load ratio, $R$, is the ratio of the minimum SIF, $K_{\min }$, and maximum SIF, $K_{\max }$, for a given cycle and is time-invariant for constant amplitude loading. The Newman closure term is denoted by $f, \Delta K$ is the SIF range defined as $K_{\max }-K_{\min }$, $\Delta K_{t h}$ is the threshold SIF range below which no growth occurs, and $K_{C}$ is the fracture toughness. The parameter values used in the present work can be found in the Appendix. The crack growth simulation was considered ground truth in place of experimental results and was used to provide simulated measurements and to quantify accuracy when investigating the proposed hypothesis.

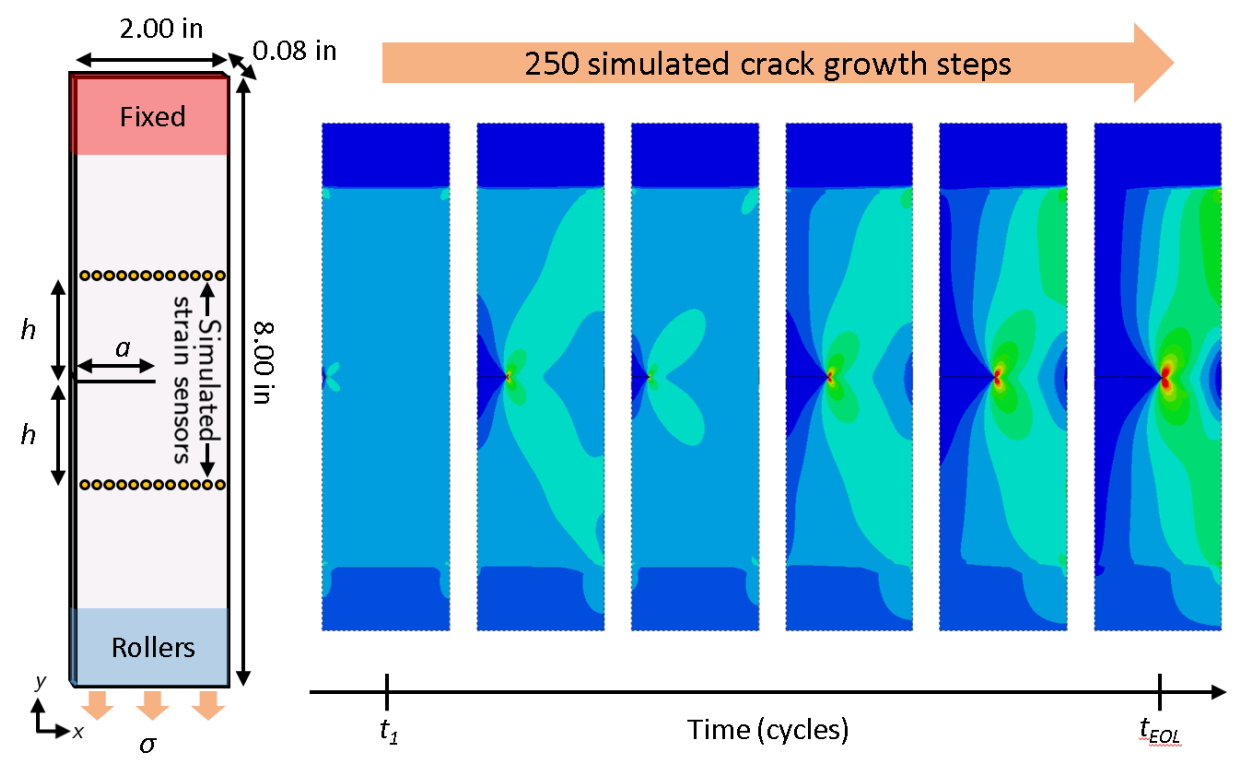

Figure 2. Illustration of the numerical example. The left diagram shows the nominal dimensions of the specimen and the applied boundary conditions where $\sigma$ is the applied stress. The fixed and roller regions imply no displacement and displacement only in the $y$ direction, respectively. The crack length, $a$, changes over time and is measured via a strain-based diagnostic technique. ${ }^{17}$ Simulated strain sensors are placed a distance $h$ from the crack, which is located at the mid-plane of the specimen. The right side of the figure is a qualitative illustration of the strain field evolution as the crack grows from the first measurement to failure. The entire simulation consisted of 250 median extension steps.

Throughout the specimen lifespan, a strain-based damage diagnosis technique ${ }^{17}$ based on Bayesian inference was used to estimate the crack length with quantified uncertainty (i.e., $q=a$ in Equation 2). Strains were extracted as shown in Figure 2 along two rows of simulated sensors, parallel to the direction of crack growth and at a distance, $h=0.93$ in. For the purposes of this study, the simulated measurements were augmented with Gaussian white noise according to a signal to noise ratio, SNR $=20$. The prognosis framework was a simplified version of an approach ${ }^{18}$ that utilized Bayesian inference and the same NASGRO (version 4) crack growth rate equation (Equation 4) used in the FE-based simulation. In contrast, however, the crack growth rate equation was solved directly as a one-dimensional approximation of the three-dimensional crack growth model. To improve this approximation and reduce sources of error that might interfere with the study, the mean SIFs obtained from each crack step of the FE-based simulation were placed in a lookup table. A linear interpolator was then used when numerically solving the ordinary differential equation (Equation 4), which is a function of $a$ and $t$, noting that $\Delta K$ is a function of $a, R$, and $\sigma$.

A set of diagnoses was required in order to obtain the first prognosis and initialize the diagnosis-prognosis feedback loop. Throughout the crack growth simulation, diagnoses were made at the fixed median extension intervals defined during the fracture simulation and corresponding to the set $\mathcal{T}_{D}=\left\{t_{3}, \ldots, t_{p}\right\}$ where $t_{p}=t_{27}$. Times $t_{1}$ and $t_{2}$ were discarded. For simplicity, each probabilistic diagnosis was dimensionally reduced to a single mean crack length in this study and placed in the set $\mathbb{D}_{t_{p}}$. At time $t_{p}, \mathbb{D}_{t_{p}}$ was used to predict the 
distribution of crack lengths for the set of times $\mathcal{T}_{P}=\left\{t_{28}, \ldots, t_{39}\right\}$. These distributions were passed to the diagnostic framework as prior knowledge for the subsequent diagnoses at all times $t \in \mathcal{T}_{P}$. The diagnosis results with and without the prognosis feedback were investigated to quantify the effectiveness of the method.

\section{Results \& Discussion}

Figure 3 shows the prognosis results generated for $\mathcal{T}_{D} \cup \mathcal{T}_{P}$ based on diagnoses from $\mathcal{T}_{D}$ only. The prognosis output consists of a mean prediction (dashed black line) and the $95 \%$ credible and prediction intervals, all of which can be compared to the ground truth curve obtained from the numerical crack growth simulation. The diagnosis data (i.e., mean crack lengths) obtained for all $t \in \mathcal{T}_{\mathcal{D}}$ are plotted as white circles. At times $t \in \mathcal{T}_{P}$, pairs of diagnoses were made corresponding to those with and without feedback. These diagnoses retained their full distributions, but only the means are plotted in Figure 3(a) for clarity. Figure 3(b) zooms in on $\mathcal{T}_{P}$ and represents the diagnosed crack length distributions as error bars corresponding to the bounds that contain $\sim 95 \%$ of the distribution's probability.

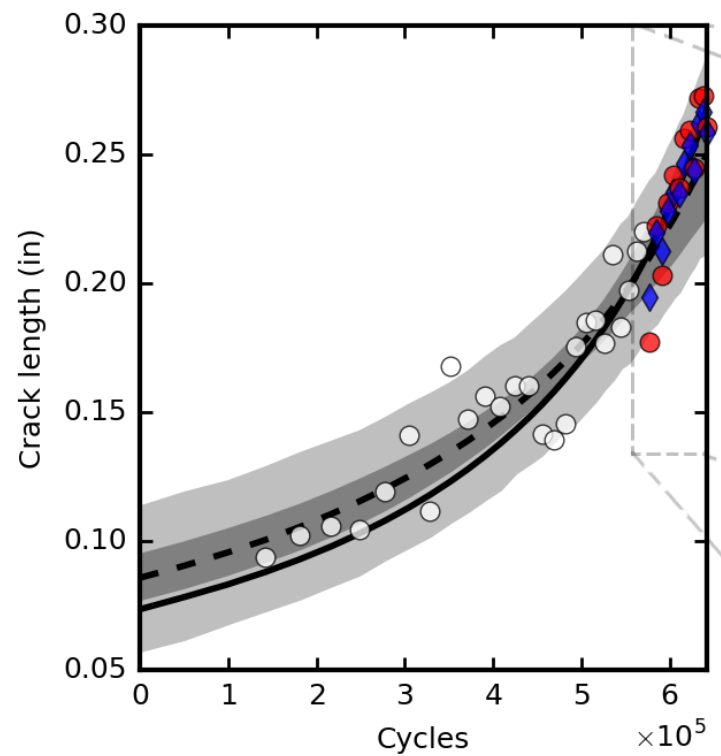

(a)

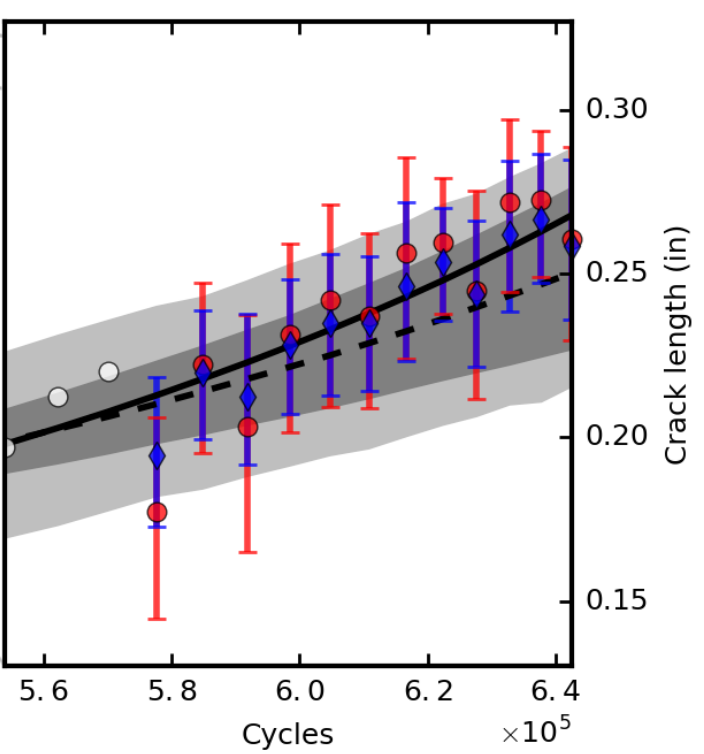

(b)

$$
\begin{aligned}
& \text { - Ground truth } \\
& \text { - } \text { Prognosis mean } \\
& 95 \% \text { credible intervals } \\
& \text { 95\% prediction intervals }
\end{aligned}
$$

Figure 3. Prognosis and diagnosis results overlain on the ground truth crack growth curve (solid black line). Diagnoses up to time $t_{p}$ used to generate the prognosis are represented by white circles. The prognosis includes a mean prediction (dashed line) as well as $95 \%$ credible and $95 \%$ prediction intervals. Sub-figure (a) only shows the mean crack lengths generated by the diagnosis framework for clarity. Sub-figure (b) is a zoomed view of the times after $t_{p}$ at which feedback was used and includes the $\mathbf{9 5 \%}$ probability bounds on the diagnosis distributions to demonstrate the effect of the feedback on uncertainty.

From Figure 3(a), the prognosis is accurate in the short term, evidenced by the fact that the true crack lengths all lie within the credible interval for $\mathcal{T}_{P}$. The prediction interval encompasses $\sim 95 \%$ of the data, as expected. Additionally, the diagnoses with feedback from this prognosis are generally more accurate than those without as they tend to be focused more around the true crack length than the diagnoses with noninformative prior distributions. Examining Figure 3(b), the effect on the means becomes clearer. At all times, the prognosis-supplied prior distribution tends to pull the mean of the uninformed diagnosis toward the prognosis mean value. As a result, if the uninformed diagnosis mean lies between the prognosis mean and the true crack length for a given time, the resulting informed diagnosis mean is less accurate, albeit by a small amount since the prior and the likelihood distributions are similar in these cases. The interaction between the noninformative (i.e., the likelihood) and the informative prior distributions is illustrated more 
clearly when examining the full marginal probability density functions (PDF) of the diagnoses and prognosis at times $t \in \mathcal{T}_{P}$, as shown in Figure 4. For all values of $\tau$ investigated, when the uninformed diagnosis mean lies outside the region bounded by the prognosis mean and the true value, accuracy is increased.

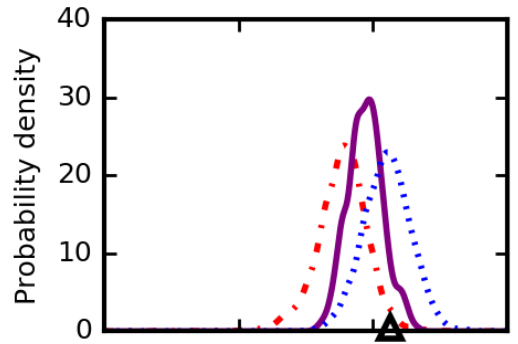

(a)

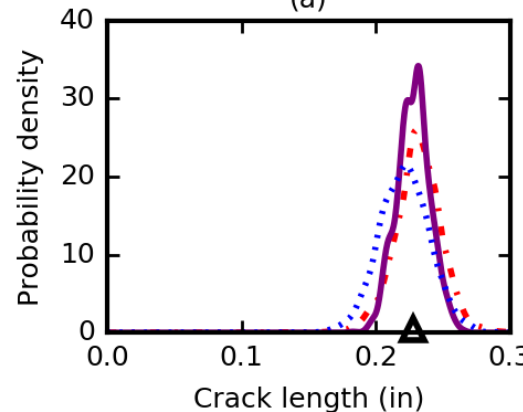

(d)

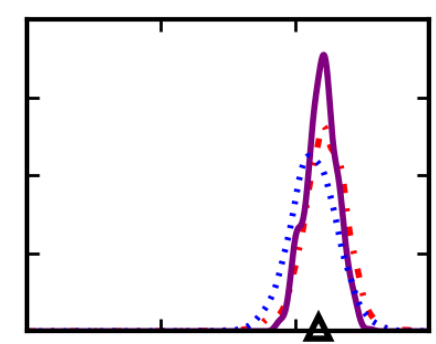

(b)

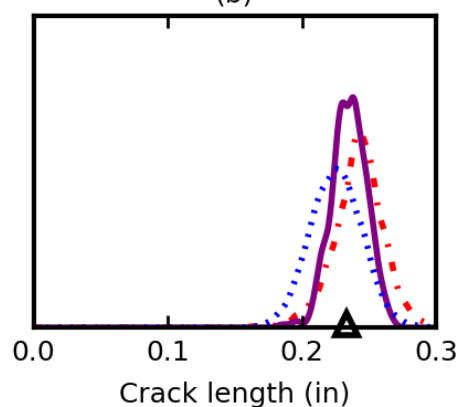

(e)

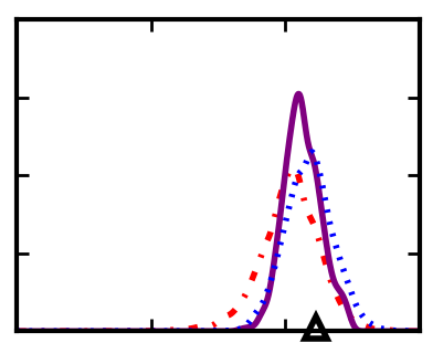

(c)

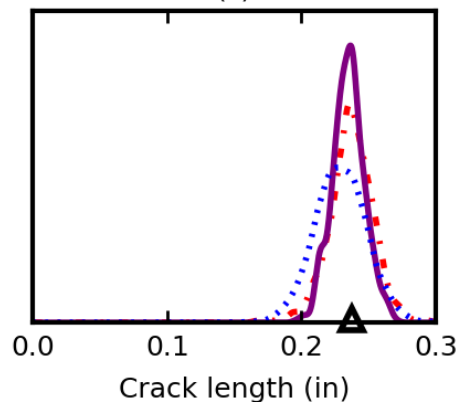

(f)

$$
\begin{array}{|llll|}
\hline-\cdots \text { Diagnosis (uninformed prior) } & \cdots \cdots & \text { Prognosis at cycle } \\
- \text { Diagnosis (prior from prognosis) } & \boldsymbol{\Delta} & \text { True crack length at cycle } \\
\hline
\end{array}
$$

Figure 4. Probability density functions (PDF) for the first six diagnoses made at $t=(\mathbf{a}) \mathbf{5 7 7 , 6 5 0}$, (b) 584,848, (c) 591,784.0, (d) 598,410, (e) 604,684 and (f) 610,812 cycles. For each time, the uninformed diagnosis and feedbackinformed diagnosis are shown along with the prognosis made at $t_{p}$, which was used to generate prior distributions for the feedback case.

Accuracy was only the first metric to be investigated; a reduction in diagnosis uncertainty was also desirable. Figure 3(b) and Figure 4 both show that the uncertainty is decreased in all cases where feedback is incorporated. This is indicated in the former by smaller error bars, meaning that the region of $95 \%$ probability is more concentrated than is the case for the uninformed diagnosis. In the latter figure, the reduction in uncertainty is represented by a decrease in width and increase in height of the crack length PDFs. All of these comparisons are quantified in Table 1 along with measures of accuracy. Here, the percent error between the true crack length, $a_{\text {true }}(t)$, and the mean diagnosis for all $t \in \mathcal{T}_{P}$ was calculated as

$$
\% \text { Error }=\frac{a_{\text {true }}(t)-d(t)}{a_{\text {true }}(t)},
$$

where $d(t)$ is the mean crack length obtained from the diagnosis at time $t$. Similarly, the reduction in the width of the $95 \%$ probability bounds was calculated as

$$
\% \text { Change }=\frac{w_{\mathrm{F}}(t)-w_{\mathrm{NI}}(t)}{w_{\mathrm{NI}}(t)},
$$

where $w_{\mathrm{F}}(t)$ and $w_{\mathrm{NI}}(t)$ indicate the width of the $95 \%$ probability bounds for the feedback and uninformed cases, respectively. For the present example, the addition of prognosis feedback resulted in a decrease in diagnosis uncertainty. Accuracy was generally improved except for the special cases where the diagnosis was already in fairly good agreement with the prior distribution provided from the prognosis. For cases where a decrease in accuracy was observed, the decrease was negligible and could be an artifact of sample size. Despite the promising performance shown, the results are necessary but not sufficient to prove the diagnosis-prognosis feedback hypothesis. Care was taken to isolate the effect of the feedback, but more work will be required to ensure that the diagnosis improvement was not based on other factors.

Furthermore, it is important to address the potential re-use of data, which would violate tenets of the underlying Bayesian analysis. It was assumed herein that, since the information from prognosis was only 
Table 1. Quantitative effect of the stochastic feedback loop. For the set $\mathcal{T}_{P}$, the percent error between the mean crack length provided by the diagnoses and the true crack length is given for the noninformative prior distributions and informative prior distributions (i.e., from feedback), respectively. The better performance at each time is highlighted with bold font. The percent change in the spread of the diagnosis distribution induced by the inclusion of the prognosis feedback relative to the diagnoses without feedback is also presented. Here, a negative sign means that the uncertainty has decreased via feedback.

\begin{tabular}{cccc}
$\begin{array}{c}\text { Time relative } \\
\text { to } t_{p}\end{array}$ & $\begin{array}{c}\text { Percent error in mean } \\
\text { diagnosis }(\text { noninformative) }\end{array}$ & $\begin{array}{c}\text { Percent error in mean } \\
\text { diagnosis (feedback) }\end{array}$ & $\begin{array}{c}\text { Percent change in } 95 \% \\
\text { probability bound width }\end{array}$ \\
\hline$t_{p+1}$ & 16.70 & $\mathbf{8 . 6 0}$ & -25.66 \\
$t_{p+2}$ & -1.95 & $\mathbf{- 0 . 8 2}$ & -24.97 \\
$t_{p+3}$ & 8.81 & $\mathbf{4 . 6 4}$ & -36.43 \\
$t_{p+4}$ & -1.47 & $\mathbf{- 0 . 0 9}$ & -28.37 \\
$t_{p+5}$ & -3.92 & $\mathbf{- 0 . 9 5}$ & -29.87 \\
$t_{p+6}$ & $\mathbf{0 . 3 9}$ & 1.24 & -22.86 \\
$t_{p+7}$ & -5.52 & $\mathbf{- 1 . 3 7}$ & -20.91 \\
$t_{p+8}$ & $\mathbf{- 4 . 6 3}$ & $\mathbf{- 2 . 3 6}$ & -16.97 \\
$t_{p+9}$ & $\mathbf{3 . 2 5}$ & 3.58 & -29.68 \\
$t_{p+10}$ & $\mathbf{- 5 . 4 2}$ & $\mathbf{- 1 . 6 1}$ & -12.90 \\
$t_{p+11}$ & $\mathbf{- 3 . 7 1}$ & $\mathbf{- 1 . 3 6}$ & -12.30 \\
$t_{p+12}$ & $\mathbf{2 . 6 9}$ & 3.50 & -17.28 \\
\hline
\end{tabular}

reused in the form of prior knowledge, there was little difference between the automated feedback and any given expert opinion, which is a typical source of prior information. Additionally, the original data gathered from the numerical simulation were strains, and there is no re-use of these strain values except what is processed and propagated forward through the prognostic framework. The feedback itself, in this case, consisted of a crack length distribution and no stress or strain information. The authors argue that this is enough, especially when coupled with the demonstrated improvements in both accuracy and uncertainty, to warrant further investigation into the approach.

\section{Concluding Remarks}

Traditionally, the relationship between damage diagnosis and prognosis is unidirectional. An alternative approach was investigated herein where diagnosis and prognosis are instead related through a stochastic feedback loop. Prognosis in the structural health management or digital twin context involves simulating damage evolution from the current state to failure. If probabilistic in nature, model output in the form of damage state distributions are available at the times between the current state and failure. These times correspond to future diagnoses, and, as such, these predictive damage state distributions can be used as prior distributions in a Bayesian-based diagnostic framework. To test the feasibility of this concept, a numerical fatigue crack growth simulation was conducted. The diagnosis-prognosis feedback loop was implemented, and diagnosis results with and without the prognosis-generated feedback were compared. In general and for all times tested, the feedback loop resulted in a sizable reduction in uncertainty as well as either an increase in accuracy or a negligible reduction in accuracy (i.e., no significant change).

The results obtained in the study are promising and support future research into the concept of a diagnosis-prognosis feedback loop. Many avenues remain open to investigation, and future work should include a deeper look into whether or not the potential re-use of data violates any aspect of the underlying Bayesian analyses. Care was taken in this study to limit noise levels and ensure a quality prognosis, resulting in an ideal case. The next step in this research will be to begin searching for degenerate cases where the feedback approach not only fails to improve the results but also degrades them (i.e., significantly decreases accuracy or increases uncertainty). While not necessarily classified as a degenerate case, there may be a time horizon beyond which prognosis results are no longer beneficial depending on the uncertainty in the prediction; this problem could potentially be alleviated by continuously updating the prognosis as often as possible. More importantly, as with any feedback loop, divergence is an issue that should not be taken lightly. Cases which cause instability or divergence should be identified if they exist. Finally, other forms of 
the diagnosis-prognosis feedback loop should be investigated, such as those that could be applied directly to the measurement process. Defining the limits of the proposed feedback approach as well as exploring new ways in which it can be applied could yield significant benefits for both structural health management and digital twin.

\section{Appendix}

The results presented herein were dependent on a numerical crack growth example that served as the ground truth for gathering simulated data and evaluating accuracy of the proposed approach. The initial finite element model mesh used for this simulation comprised 3,699 Tet10 elements and 7,024 total nodes. The analysis was completed using the implicit version of the parallel finite element code ScIFEN. ${ }^{14}$ Remeshing was conducted within FRANC3D ${ }^{15}$ upon crack insertion and at every growth step. The mesh size was then dependent on the length of the crack and the crack front template radius, which is a special ring of elements placed around the crack front. In this study, the template radius was $0.002 \mathrm{in}$. The elastic modulus and Poisson's ratio used for aluminum alloy 2024-T3 were 10,600 ksi and 0.33, respectively.

The NASGRO parameters used to model the crack growth rate in the numerical simulation are presented in Table 2. The NASGRO equation (Equation 4) can be fully defined with these parameters, and is a function of only crack length, $a$, and time, $t$, measured in fatigue cycles. The dependence on $a$ is introduced via the stress intensity factor (SIF) range, $\Delta K$ where $\Delta K=K_{\max }-K_{\min } . K_{\max }$ and $K_{\min }$ are the maximum and minimum SIFs at each cycle, respectively, and depend on the crack length, $a$, and the applied load. Some of the parameters are functions of more fundamental material properties; in that case, the equations used to calculate them are provided. The load ratio, $R$, is the ratio of the minimum and maximum SIFs for a

Table 2. Parameters used in the NASGRO equation (Equation 4).

\begin{tabular}{ccl} 
Parameter & Value & Equation \\
\hline$C$ & $8.0 \times 10^{-9}$ & - \\
$n$ & 3.20 & - \\
$p$ & 0.25 & - \\
$q$ & 1.00 & - \\
$R$ & 0.10 & $K_{\min } / K_{\max }$ \\
$\Delta K_{t h}$ & - & $\Delta K_{1}^{*}\left[\frac{1-R}{1-f}\right]^{\left(1+R C_{t h}^{+}\right)} /\left(1-A_{0}\right)^{(1-R) C_{t h}^{+}}$ \\
$\Delta K_{1}^{*}$ & - & $\Delta K_{1}\left[\frac{a}{a+a_{0}}\right]^{1 / 2}$ \\
$a_{0}$ & $1.5 \times 10^{-3}$ in & - \\
$C_{t h}^{+}$ & 1.21 & - \\
$\Delta K_{1}$ & $1.22 \mathrm{ksi} \sqrt{\text { in }}$ & - \\
$f$ & - & $\max \left(R, A_{0}+A_{1} R+A_{2} R^{2}+A_{3} R^{3}\right)$ \\
$A_{0}$ & - & $\left(0.825-0.34 \alpha+0.05 \alpha^{2}\right) \times\left[\cos \left(\frac{\pi}{2} \frac{S_{m a x}}{\sigma_{0}}\right)\right]^{1 / \alpha}$ \\
$A_{1}$ & - & $(0.415-0.071 \alpha) \frac{S_{m a x}}{\sigma_{0}}$ \\
$A_{2}$ & - & $1-A_{0}-A_{1}-A_{3}$ \\
$A_{3}$ & - & $2 A_{0}+A_{1}-1$ \\
$\frac{S_{m a x}}{\sigma_{0}}$ & 0.30 & - \\
$\alpha$ & 2.00 & - \\
$K_{c}$ & - & $K_{I C}\left(1+B_{k} e^{\left.-A_{k} t / t_{0}\right)^{2}}\right.$ \\
$K_{I C}$ & $30.0 \mathrm{ksi} \sqrt{\text { in }}$ & - \\
$A_{k}$ & 1.00 & - \\
$B_{k}$ & 1.50 & - \\
$t_{0}$ & - & $2.5\left(K_{I C} / \sigma_{Y}\right)^{2}$ \\
$\sigma_{Y}$ & $53.0 \mathrm{ksi}$ & - \\
$t$ & $0.08 \mathrm{in}$ & - \\
\hline & &
\end{tabular}


given cycle ( $R$ is constant for the simulations presented herein), $f$ is the Newman closure term, $\Delta K_{t h}$ is the threshold SIF range below which no growth occurs, $\Delta K_{1}$ is the observed threshhold for a high $R$ value, $K_{C}$ is the fracture toughness, $K_{I C}$ is the plane strain fracture toughness, $\alpha$ is a plane stress/strain constraint factor, $\frac{S_{\max }}{\sigma_{0}}$ is the ratio of maximum applied stress to flow stress, $t$ is the approximate thickness of the specimen, $\sigma_{Y}$ is the material yield stress, and $a_{0}$ is a small crack parameter. The remaining parameters are empirical constants.

\section{References}

${ }^{1}$ Chang, F.-K., Markmiller, J. F., Ihn, J.-B., and Cheng, K. Y., "A potential link from damage diagnostics to health prognostics of composites through built-in sensors," J. Vib. Acoust., Vol. 129, No. 6, 2007, pp. 718-729.

${ }^{2}$ Ling, Y. and Mahadevan, S., "Integration of structural health monitoring and fatigue damage prognosis," Mech. Syst. Sig. Process., Vol. 28, 2012, pp. 89-104.

${ }^{3}$ Farrar, C. R. and Lieven, N. A., "Damage prognosis: the future of structural health monitoring," Philos. Trans. R. Soc. London, Ser. A, Vol. 365, No. 1851, 2007, pp. 623-632.

${ }^{4}$ Sankararaman, S., Ling, Y., Shantz, C., and Mahadevan, S., "Uncertainty quantification in fatigue crack growth prognosis," Int. J. Prog. Health Manage., Vol. 2, No. 1, 2011, pp. 1-15.

${ }^{5}$ Sankararaman, S., Ling, Y., and Mahadevan, S., "Uncertainty quantification and model validation of fatigue crack growth prediction," Eng. Fract. Mech., Vol. 78, No. 7, 2011, pp. 1487-1504.

${ }^{6}$ Peng, T., He, J., Xiang, Y., Liu, Y., Saxena, A., Celaya, J., and Goebel, K., "Probabilistic fatigue damage prognosis of lap joint using Bayesian updating," J. Intell. Mater. Syst. Struct., Vol. 26, No. 8, 2015, pp. 965-979

${ }^{7}$ Chiachıo, J., Chiachıo, M., Saxena, A., Rus, G., and Goebel, K., "An energy-based prognostics framework to predict fatigue damage evolution in composites," Proceedings of the Annual Conference of the Prognostics and Health Management Society, PHM Society, New Orleans, Louisiana, USA, 14-17 October, 2013, pp. 363-371.

${ }^{8} \mathrm{Su}, \mathrm{Z}$., Ye, L., and Lu, Y., "Guided Lamb waves for identification of damage in composite structures: A review," J. Sound Vib., Vol. 295, No. 3, 2006, pp. 753-780.

${ }^{9} \mathrm{Ihn}$, J.-B. and Chang, F.-K., "Detection and monitoring of hidden fatigue crack growth using a built-in piezoelectric sensor/actuator network: I. Diagnostics," Smart Mater. Struct., Vol. 13, No. 3, 2004, pp. 609.

${ }^{10}$ Barthorpe, R. J., On Model- and Data-Based Approaches to Structural Health Monitoring, Ph.D. thesis, University of Sheffield, 2010

${ }^{11}$ Tikhonov, A. N. and Arsenin, V. Y., Solution of Ill-Posed Problems, Halsted, New York, 1979.

${ }^{12}$ Smith, R. C., Uncertainty Quantification: Theory, Implementation, and Applications, SIAM, Philadelphia, 2013.

${ }^{13}$ Aster, R. C., Borcers, B., and Thurber, C. H., Parameter Estimation and Inverse Problems, Academic Press, Waltham, 2013.

${ }^{14}$ Warner, J. E., Bomarito, G. B., Heber, G., and Hochhalter, J. D., "Scalable implementation of finite elements by NASA - implicit (ScIFEi)," NASA/TM-2016-219180, 2016.

${ }^{15}$ Fracture Analysis Consultants Inc, FRANC3D Reference Manual, Version 6, 2011.

${ }^{16}$ NASA Johnson Space Center and Southwest Research Institute, NASGRO: Fracture Mechanics and Fatigue Crack Growth Analysis Software Reference Manual, Version 4.01, 2002.

${ }^{17}$ Warner, J., Hochhalter, J., Leser, W., Leser, P., and Newman, J., "A computationally-efficient inverse approach to probabilistic strain-based damage diagnosis," Proceedings of the Annual Conference of the Prognostics and Health Management Society, PHM Society, Denver, Colorado, USA, 3-6 October, 2016, pp. 1-15.

${ }^{18}$ Leser, P., Newman, J., Warner, J., Leser, W., Hochhalter, J., and Yuan, F.-G., "Probabilistic prognosis of non-planar fatigue crack growth," Proceedings of the Annual Conference of the Prognostics and Health Management Society, PHM Society, Denver, Colorado, USA, 3-6 October, 2016, pp. 1-15. 